\title{
Six-Minute Walk Distance Is an Independent Predictor of Hospital Readmission in Patients With Chronic Heart Failure
}

\author{
Minoru Taвata, ${ }^{1}$ MS, Ryosuke Shimizu, ${ }^{1}$ MS, Daisuke Kamekawa, ${ }^{1}$ MS, Michitaka Kato, ${ }^{1}$ MS, \\ Kentaro KamiYa, ${ }^{1}$ MS, Ayako AkiYama, ${ }^{1}$ MS, Yumi Kamada, ${ }^{1}$ MS, Shinya Tanaka, ${ }^{1}$ MS, \\ Chiharu NodA, ${ }^{3} \mathrm{MD}$, and Takashi MASUdA, ${ }^{1,2} \mathrm{MD}$
}

\begin{abstract}
SUMMARY
Patients with chronic heart failure $(\mathrm{CHF})$ are frequently readmitted to the hospital due to disease progression. Although a shorter 6-minute walk distance (6MWD) is correlated with poor prognosis, 6MWD is not considered a clinical indicator for predicting hospital readmission.

We investigated whether 6MWD measured at the time of hospital discharge predicted readmission due to heart failure in CHF patients.

Patients admitted to the hospital for the first time due to heart failure were enrolled. After 6MWD was measured at discharge, patients were followed-up for 3 years. Clinical characteristics, 6MWD and readmission due to heart failure were evaluated in 252 patients $(68.5 \pm 11.8$ years old, 162 males). Significant factors that affected readmission were extracted and cut-off values were determined using multivariate logistic regression analysis and receiver operating characteristic curves.

Of $252 \mathrm{CHF}$ patients, 103 were readmitted within 3 years. 6MWD at the time of discharge was significantly shorter in readmitted patients than non-readmitted patients $(P<0.001)$ and was a significant predictor of readmission $(P<$ $0.001)$. The odds ratio for readmission was $1.22(P<0.001)$ with each 10 -meter decrease in $6 \mathrm{MWD}$. The 6MWD cut-off value was determined to be 390 meters, with a sensitivity of 0.75 and a specificity of 0.77 .

6MWD measured at the time of discharge is an independent predictor of hospital readmission in CHF patients, with a cut-off value of 390 meters. (Int Heart J 2014; 55: 331-336)
\end{abstract}

Key words: Exercise capacity, Cardiac rehabilitation

$\mathrm{P}$ atients with chronic heart failure (CHF) are frequently readmitted to the hospital and have a high mortality rate due to disease progression. ${ }^{1-4)} \mathrm{CHF}$ patients also have a lower exercise capacity. ${ }^{5}$ Six-minute walk distance (6MWD) measurements are often performed to assess exercise capacity. ${ }^{6}$ Many studies have shown an increase in mortality and morbidity in CHF patients with a $6 \mathrm{MWD}<300$ meters. ${ }^{6,7}$ The mortality rate was reported to be 4.6-fold higher in CHF patients with a $6 \mathrm{MWD}<218$ meters than in those with a $6 \mathrm{MWD} \geq 218$ meters. ${ }^{7} 6 \mathrm{MWD}$ is often used as a clinical indicator of prognosis in CHF patients. In a study evaluating hospital readmission due to disease progression, the readmission rate was reported to be significantly higher in CHF patients with a $6 \mathrm{MWD}<200$ meters compared to patients with a $6 \mathrm{MWD} \geq 200$ meters. ${ }^{8}$ In contrast, Roul, et al did not observe a significant relationship between readmission and 6MWD in CHF patients, although peak oxygen uptake was negatively correlated with the readmission rate. ${ }^{5}$ Applicability of 6MWD as a clinical indicator to predict hospital readmission in CHF patients has not been determined.
This study investigated whether 6MWD measured at the time of hospital discharge predicted readmission due to disease progression in CHF patients after their first heart failure-related hospitalization. In addition, we attempted to establish a 6MWD cut-off value to predict readmission, if a 6MWD was a significant limiting factor for readmission.

\section{Methods}

Patients: The study was approved by the Ethics Committee on Human Research at Kitasato University and performed in accordance with the Declaration of Helsinki. Procedures and possible risks of the study were clearly explained, and patients provided written informed consent prior to participation.

CHF patients admitted to the Cardiovascular Center at Kitasato University Hospital between September 1, 2005 and August 31, 2009 for the first time due to heart failure were enrolled in the study. Patients with a New York Heart Association (NYHA) functional classification of III or IV were eligible to

From the ${ }^{1}$ Department of Cardiovascular Medicine, Kitasato University Graduate School of Medical Sciences, ${ }^{2}$ Department of Rehabilitation, School of Allied Health Sciences, Kitasato University, and ${ }^{3}$ Department of Cardiovascular Medicine, Kitasato University School of Medicine, Sagamihara, Japan.

Address for correspondence: Takashi Masuda, MD, Department of Rehabilitation, School of Allied Health Sciences, Kitasato University, 1-15-1 Kitasato, Minami-ku, Sagamihara, Kanagawa, 252-0373 Japan. E-mail: tak9999@med.kitasato-u.ac.jp

Received for publication August 6, 2013. Revised and accepted January 10, 2014.

Released in advance online on J-STAGE June 5, 2014.

All rights reserved by the International Heart Journal Association. 
participate. Patients with de novo heart failure due to acute cardiovascular events or on regular hemodialysis or patients with cognitive impairments or walking disabilities caused by neurological or orthopedic disorders were excluded from the study. According to a report from the Japanese Cardiac Registry of Heart Failure in General Practice (JCARE-GENERAL), hospital readmission occurred within 30 months from initial hospital discharge in the majority of CHF patients. ${ }^{9)}$ Therefore, patients were prospectively followed-up for 3 years from the discharge, after they received cardiac rehabilitation during initial hospitalization and 6MWD measurement at the time of discharge. Patients who were readmitted to the hospital for reasons other than heart failure within the follow-up period were excluded. A total of $252 \mathrm{CHF}$ patients (162 males and 90 females) were enrolled in this study.

As patient characteristics, age, gender, causes of $\mathrm{CHF}$, plasma brain natriuretic peptide (BNP), left ventricular ejection fraction (LVEF) measured using an echocardiogram at admission, NYHA functional classification at admission and discharge, and duration of hospitalization were obtained from medical records.

Cardiac rehabilitation: In our hospital, patients received cardiac rehabilitation during the hospitalization according to the guideline of rehabilitation for cardiovascular disease published by the Japanese Circulation Society. ${ }^{10)}$ Exercise therapy of the rehabilitation consisted of sitting on a chair and walking 10 , 50, 100, 200 and 500 meters in a ward, the workload of which was gradually increased after the beginning of rehabilitation.

Six-minute walk distance: To assess the exercise capacity of CHF patients, 6MWD measurements were obtained at the time of hospital discharge in accordance with guidelines established by the American Thoracic Society. ${ }^{11)}$ Before measuring $6 \mathrm{MWD}$, a physical therapist explained the procedure to the patients. Patients were instructed to rest in a sitting position at the starting line for 10 minutes without a warm up. They were told to walk as fast and long as possible on a 30-meter walkway that was marked every 3 meters. Patients were allowed to stop and rest or reduce their walking speed if they felt shortness of breath or fatigue. The physical therapist encouraged the patients (eg, "you are doing well", "keep up the good work") every minute and alerted them when 15 seconds of testing remained. 6MWD measurement was suspended if patients experienced chest pain, dyspnea, leg cramps, hydrosis, cyanosis or facial pallor during the test.

Cumulative readmission rate: Readmission was defined as a second hospital admission due to worsening CHF within the 3-year follow-up period. The number of days from the first hospital discharge to readmission was determined, and the proportion of readmitted patients to total CHF patients enrolled in the study was calculated as the cumulative readmission rate. If CHF patients were admitted to the hospital more than twice within the 3-year follow-up period, the cumulative readmission rate was calculated using the number of days from the first discharge date to the second admission.

Limiting factors: Multivariate logistic regression analysis was used to extract factors that affected readmission due to heart failure based on patient characteristics, including 6MWD at discharge. Extracted factors were defined as limiting factors in the readmission of CHF patients.

Sample size: Sample size was determined using the range of permissible error and confidence interval $(\mathrm{CI}) .{ }^{12,13}$ If a signifi- cance level of CI was $95 \%$, the range of permissible error was calculated using the following formula: $2 \times$ (normal distribution $) \times \sqrt{ }$ power of test $\times \sqrt{ }(1$-power of test $) \div \sqrt{ }$ number of patients. Therefore, the number of patients fitted within the range of permissible error was obtained based on the following formula: $2^{2} \times(\text { normal distribution })^{2} \times$ power of test $\times(1$-power of test $) \div$ (permissible error $)^{2}$. ${ }^{12,13)}$

Because the power of test, significance level and permissible error were given as $0.80,0.95$ and 0.10 , respectively, a normal distribution of sample size was determined by $1.96 .{ }^{14)}$ The required number of patients was determined as follows: $2^{2}$ $\times 1.96^{2} \times 0.80 \times(1-0.80) \div 0.10^{2}=246$. Sample size was considered statistically significant, if more than 246 patients were evaluated.

Statistical analyses: Clinical characteristics were compared between male and female patients because gender is known to affect 6MWD results. ${ }^{11)}$ Differences in clinical characteristics between readmitted and non-readmitted patients were also assessed. Statistical significance was analyzed by comparing variables between male and female patients and readmitted and non-readmitted patients using the unpaired Student $t$-test or chi-square test.

Multivariate logistic regression analysis using the forward selection method with likelihood ratio was performed for age, gender, BNP, LVEF, NYHA functional classification at admission and discharge, duration of hospitalization, and 6MWD as exploratory variables and readmission as the dependent variable. ${ }^{15)}$ The logistic regression formula was determined using the limiting factors for readmission, the model fit of which was accessed by the Hosmer-Lemeshow test. Significance level, odds ratio and CI $>95 \%$ were calculated for each limiting factor. Limiting factors were analyzed using receiver operating characteristic (ROC) curves, and cut-off values were determined using the area under the ROC curve (AUC). ${ }^{16}$

Cross tabulation was established using the number of readmitted and non-readmitted patients as determined by the cutoff value for each limiting factor. Based on cross tabulation, diagnostic odds ratios, relative risk for readmission, and positive and negative likelihood ratios were calculated using the chi-square test and Fisher's exact test.

Continuous variables are expressed as the mean \pm standard deviation (SD). The significance level was set at $P>0.05$ for the model fit of the formula to predict the readmission, and $P<0.05$ for other statistical tests. Statistical analysis was performed using SPSS ver. 12.0J (SPSS, Chicago, IL, USA).

\section{ReSULTS}

Table I shows the clinical characteristics of male and female patients at the first hospital admission. The cohort consisted of 162 males and 90 females with a mean age of $68.5 \pm$ 11.3 years. Primary diseases included ischemic heart disease and cardiomyopathy, and 209 patients were considered NYHA class IV (82.9\%). LVEF at admission was significantly higher and 6MWD at discharge was significantly shorter in females compared to males ( $P<0.001$ for both). There were no significant differences in age, BNP, primary diseases, NYHA functional classification at admission and discharge, duration of hospitalization, and cumulative readmission rate between male and female patients. 
Table I. Patient Characteristics

\begin{tabular}{|c|c|c|c|c|}
\hline & Total & Male & Female & $P$ \\
\hline Number of patients & 252 & 162 & 90 & \\
\hline Age (years) & $68.5 \pm 11.3$ & $67.5 \pm 10.9$ & $70.4 \pm 11.9$ & NS \\
\hline Height (m) & $1.59 \pm 0.11$ & $1.64 \pm 0.09$ & $1.53 \pm 0.06$ & $P<0.005$ \\
\hline $\mathrm{BNP}(\mathrm{pg} / \mathrm{mL})$ & $998 \pm 831$ & $1021 \pm 809$ & $957 \pm 870$ & NS \\
\hline $\operatorname{LVEF}(\%)$ & $35.4 \pm 12.8$ & $31.9 \pm 11.6$ & $41.9 \pm 13.4$ & $P<0.001$ \\
\hline Primary diseases & & & & NS \\
\hline Ischemic heart disease & $77(30.6 \%)$ & $57(35.2 \%)$ & $20(22.2 \%)$ & \\
\hline Valvular heart disease & $50(19.8 \%)$ & $24(14.8 \%)$ & $26(28.9 \%)$ & \\
\hline Cardiomyopathy & $73(29.0 \%)$ & $55(33.9 \%)$ & $18(20.0 \%)$ & \\
\hline Hypertension & $29(11.5 \%)$ & $15(9.3 \%)$ & $14(15.6 \%)$ & \\
\hline Others & $23(9.1 \%)$ & $11(6.8 \%)$ & $12(13.3 \%)$ & \\
\hline NYHA functional classification at admission & & & & NS \\
\hline Class III & $43(17.1 \%)$ & $32(12.7 \%)$ & $11(4.4 \%)$ & \\
\hline Class IV & $209(82.9 \%)$ & $130(51.6 \%)$ & $79(31.3 \%)$ & \\
\hline \multicolumn{5}{|l|}{ NYHA functional classification at discharge } \\
\hline Class II & $218(86.5 \%)$ & $144(57.1 \%)$ & $74(29.4 \%)$ & NS \\
\hline Class III & $34(13.5 \%)$ & $18(7.1 \%)$ & $16(6.4 \%)$ & \\
\hline 6MWD (m) & $401.4 \pm 79.2$ & $418.3 \pm 75.4$ & $369.8 \pm 76.7$ & $P<0.001$ \\
\hline Duration of hospitalization (days) & $18.4 \pm 13.1$ & $17.9 \pm 12.2$ & $18.7 \pm 14.4$ & NS \\
\hline No. of readmitted patients & 103 & 64 & 39 & NS \\
\hline Cumulative readmission rate & $40.9 \%$ & $39.5 \%$ & $43.3 \%$ & NS \\
\hline
\end{tabular}

Data are presented as mean \pm SD. M indicates meters; LVEF, left ventricular ejection fraction; 6MWD, 6-minute walk distance; BNP, plasma brain natriuretic peptide; and NYHA, New York Heart Association.

Table II. Characteristics of Readmitted and Non-Readmitted Patients

\begin{tabular}{lccc}
\hline & Readmitted patients & Non-readmitted patients & $P$ \\
\hline Number of patients & 103 & 149 & \\
Male / Female & $64 / 39$ & $98 / 51$ & NS \\
Age (years) & $70.0 \pm 10.2$ & $69.6 \pm 12.0$ & NS \\
Height (m) & $1.56 \pm 0.12$ & $1.62 \pm 0.11$ & NS \\
BNP (pg/mL) & $958 \pm 655$ & $1,009 \pm 963$ & NS \\
LVEF (\%) & $34.4 \pm 11.5$ & $36.1 \pm 13.7$ & NS \\
Primary diseases & $38(36.9 \%)$ & $39(36.9 \%)$ & NS \\
$\quad$ Ischemic heart disease & $23(22.3 \%)$ & $27(18.1 \%)$ & \\
Valvular heart disease & $26(25.2 \%)$ & $47(31.5 \%)$ & \\
Cardiomyopathy & $6(5.8 \%)$ & $23(15.5 \%)$ & \\
Hypertension & $13(9.7 \%)$ & $13(8.7 \%)$ & NS \\
$\quad$ Others & $19(7.5 \%)$ & $24(9.5 \%)$ & \\
NYHA functional classification at admission & $97(38.5 \%)$ & $112(44.5 \%)$ & $P<0.05$ \\
$\quad$ Class III & $83(32.9 \%)$ & $135(53.6 \%)$ & \\
Class IV & $20(7.9 \%)$ & $14(5.6 \%)$ & \\
NYHA functional classification at discharge & $347.7 \pm 57.3$ & $437.8 \pm 70.9$ & $P<0.001$ \\
$\quad$ Class II & $19.4 \pm 16.2$ & $18.2 \pm 14.4$ & $P<0.05$ \\
$\quad$ Class III & & \\
6MWD (m) & & \\
Duration of hospitalization (days) &
\end{tabular}

Data are presented as mean $\pm \mathrm{SD}$, $\mathrm{m}$ indicates meters; LVEF, left ventricular ejection fraction; 6MWD, 6-minute walk distance; BNP, plasma brain natriuretic peptide; and NYHA, New York Heart Association.

Table II shows the clinical characteristics of readmitted and non-readmitted patients. There were 103 and 149 readmitted and non-readmitted patients, respectively. The 6MWD at discharge was significantly shorter in readmitted patients than non-readmitted patients $(P<0.001)$. NYHA functional classification at discharge was significantly worse and duration of hospitalization was significantly longer in readmitted patients than non-readmitted patients $(P<0.05$, respectively). However, there were no significant differences in gender ratio, age, height, BNP, LVEF, primary diseases, and NYHA functional classification at admission between the groups.

Figure 1 shows the cumulative readmission rate in $\mathrm{CHF}$ patients, which reached $30 \% 1$ year after the initial hospital discharge and $40 \%$ during the 3-year follow-up period. Approximately $70 \%$ of readmitted patients returned to the hospital within the first year.

Figure 2 shows the results of multivariate logistic regression analysis. 6MWD at discharge, age and LVEF were significant independent limiting factors for readmission due to heart failure. The odds ratios of readmission for each 10-meter decrease in 6MWD, each 1-year increase in age, and each $1 \%$ decrease in LVEF were 1.22 (95\% CI: 1.1-1.34, $P<0.001)$, 1.05 (95\% CI: $1.02-1.09, P<0.01)$, and 1.02 (95\% CI: $1.001-$ $1.04, P<0.05)$, respectively. 


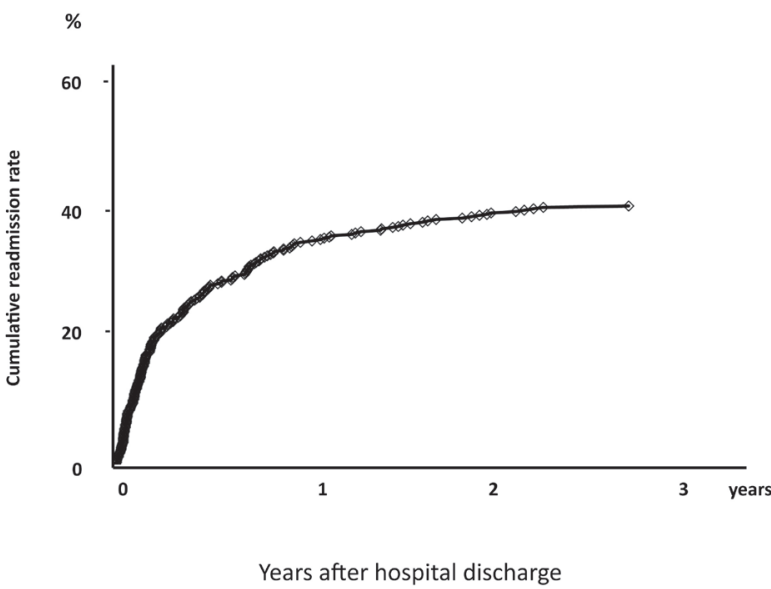

Figure 1. Cumulative readmission rate in CHF patients within 3 years of hospital discharge. CHF indicates chronic heart failure.

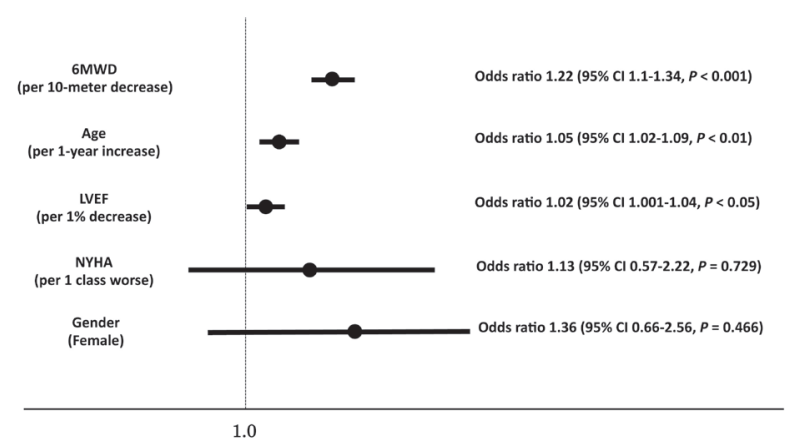

Figure 2. Significant predictors and odds ratios for readmission as assessed by multivariate logistic regression analysis. Multivariate logistic regression analysis extracted 6MWD at hospital discharge, age and LVEF as significant independent limiting factors of readmission due to CHF. CHF indicates chronic heart failure, 6MWD; 6-minute walk distance, $\mathrm{LVEF}$, left ventricular ejection fraction, NYHA; New York Heart Association; and $\mathrm{CI}$, confidence interval.

Table III. Model fit Accessed by Hosmer-Lemeshow Test

\begin{tabular}{lrcc}
\hline $\begin{array}{c}\text { Limiting factors for } \\
\text { readmission }\end{array}$ & \multicolumn{1}{c}{$\chi^{2}$} & $P$ & Predictive value \\
\hline 6MWD & 13.49 & $P=0.096$ & $74.6 \%$ \\
6MWD and age & 5.93 & $P=0.655$ & $75.4 \%$ \\
6MWD, age and LVEF & 18.82 & $P=0.016$ & $77.5 \%$ \\
\hline
\end{tabular}

6MWD indicates 6-minute walk distance and LVEF, left ventricular ejection fraction.

Table III shows the model fit and predictive accuracy of univariate and multivariate logistic regression analyses in a 6MWD, age and LVEF as limiting factors for readmission. The regression analysis formula using 6MWD at discharge and age satisfied statistical significance to predict the readmission due to CHF.

Figure 3 shows the ROC curve for 6MWD in predicting readmission due to heart failure. The AUC for 6MWD was $0.84(P<0.001)$. When the 6MWD cut-off value was set at

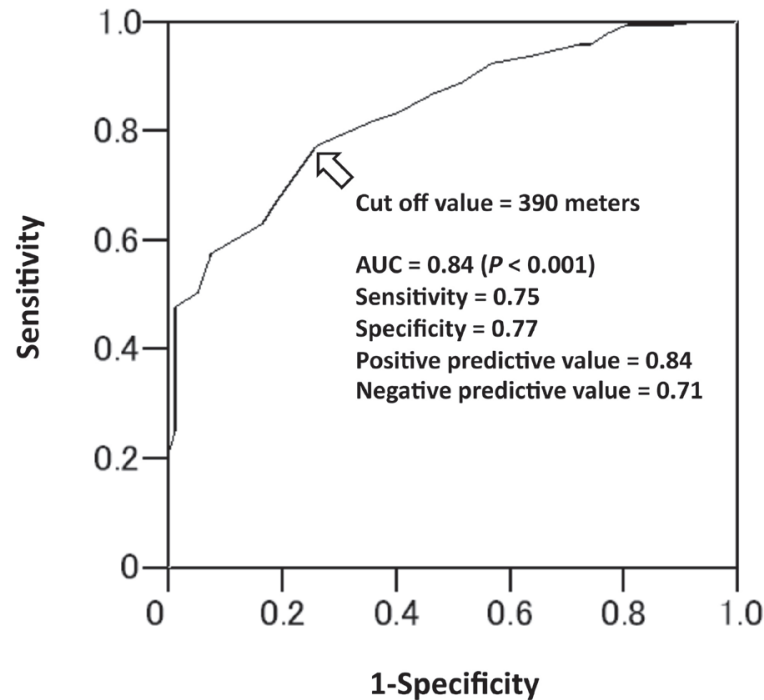

Figure 3. ROC curve to predict readmission using 6MWD. The AUC for 6MWD at discharge was 0.84 in CHF patients $(P<0.001)$, with 390 meters as the optimum $6 \mathrm{MWD}$ cut-off value. ROC indicates receiver operating characteristic; 6MWD, 6-minute walk distance; AUC, area under the ROC curve; and CHF, chronic heart failure.

Table IV. Actual Number of Readmitted and Non-Readmitted Patients Using the 6MWD Cut-Off Value

\begin{tabular}{lcc}
\hline 6 MWD cut-off value & Readmitted patients & Non-readmitted patients \\
\hline $6 \mathrm{MWD} \leqq 390$ meters & $80(31.8 \%)$ & $33(13.1 \%)$ \\
$6 \mathrm{MWD}>390$ meters & $23(9.1 \%)$ & $116(46.0 \%)$ \\
\hline
\end{tabular}

$\chi^{2}=66.24(P<0.001) .6 \mathrm{MWD}$ indicates 6-minute walk distance.

390 meters, the sensitivity and specificity were 0.75 and 0.77 , respectively. AUCs for age (0.43) and LVEF (0.52) were not statistically significant.

Table IV shows the actual number of readmitted and nonreadmitted patients based on a 6MWD cut-off value of 390 meters. Diagnostic odds ratio and relative risk for readmission were 12.23 (95\% CI: 6.7-22.3, $P<0.001)$ and 2.86 (95\% CI: 2.23-3.64), respectively. Positive and negative predictive values were 0.84 (95\% CI: 0.79-0.88) and 0.71 (95\% CI: 0.65-0.76), respectively, and positive and negative likelihood ratios were 3.49 (95\% CI: 2.53-4.89) and 0.29 (95\% CI: 0.22-0.38), respectively. Because the relative risk, positive and negative predictive values, and positive and negative likelihood ratios were statistically significant, 6MWD at discharge was considered a significant predictor for hospital readmission due to heart failure.

\section{Discussion}

This is the first report suggesting a 6MWD cut-off value of 390 meters to predict the likelihood of patient readmission. This study demonstrated that 6MWD measured at the time of hospital discharge is a significant independent clinical indicator in predicting readmission in patients with worsening $\mathrm{CHF}$.

6MWD has been used to evaluate motor function and ex- 
ercise capacity in CHF patients. ${ }^{17)}$ 6MWD is considered highly reliable, even if it is only measured once, because it has an interclass correlation coefficient of 0.88 (95\% CI: 0.79-0.94). ${ }^{18)}$ American Thoracic Society guidelines state that 6MWD is an indicator of cardiopulmonary function and muscular strength and is a comprehensive indicator for functional capacity in the activities of daily living. ${ }^{11)}$ 6MWD can be safely and simply performed in CHF patients ${ }^{17,19-22)}$ and is considered a suitable method of assessing functional capacity in patients who cannot undergo exercise testing using a treadmill or cycle ergometer.

6MWD has been reported to be influenced by gender. ${ }^{11)}$ Therefore, we investigated gender differences and clinical characteristics. LVEF was significantly higher and 6MWD at discharge was shorter in females than in males. Previous studies have reported no gender differences in the readmission rate of CHF patients, although LVEF on admission was shown to be higher in females than males. ${ }^{23)}$ The present study found no significant difference in the readmission rate between males and females. Gender differences in 6MWD have been attributed to differences in height rather than motor function or exercise capacity. ${ }^{23)}$ It is unlikely that gender difference affects the prediction of readmission using 6MWD.

The present study showed that 6MWD was significantly shorter in readmitted versus non-readmitted patients and confirmed a previous report stating that $\mathrm{CHF}$ patients with a shorter 6MWD had a higher readmission rate. ${ }^{5,7)}$ 6MWD at discharge, age and LVEF were independent limiting factors in CHF patient readmission.

ROC analysis indicated that a 6MWD cut-off value of 390 meters at discharge can predict readmission based on an AUC of 0.84 , which indicates high predictive accuracy. These analyses strongly suggest that 6MWD is a highly sensitive, specific predictor of readmission due to heart failure. However, ROC analysis did not detect optimal cut-off values for age and LVEF in predicting readmission, suggesting that the readmission rate is positively correlated with age and negatively correlated with LVEF. If the 6MWD is $<390$ meters at the time of discharge, the relative risk of readmission is 2.86-fold higher than that in patients with a $6 \mathrm{MWD} \geq 390$ meters. Therefore, 6MWD measured at the time of discharge is considered a useful, specific predictor of readmission due to heart failure. Likelihood of readmission can be determined using a 6MWD cutoff value of 390 meters.

Limitations: 6MWD results are influenced by leg strength and balance in CHF patients. ${ }^{24,25)}$ However, leg strength and balance were not assessed at the time of hospital discharge in the present study. 6MWD measured at discharge may be shorter in readmitted patients if their leg strength or balance was poor or compromised at the initial hospital admission. In addition, we did not fully consider drug compliance, which is known to be a limiting factor in readmission due to heart failure. $^{26)}$ The readmission rate might be increased if patients with poor drug compliance participated in the present study. Further studies are needed to elucidate the relationship between these factors and 6MWD.

Conclusions: 6MWD measured at the time of hospital discharge was determined to be an independent predictor of readmission in patients with worsening CHF, with a 6MWD cutoff value of 390 meters. Because CHF patients with a 6MWD $<390$ meters at discharge had a high readmission rate, 6MWD is considered a strong clinical indicator for hospital readmis- sion due to heart failure.

\section{REFERENCES}

1. Fonarow GC, Abraham WT, Albert NM, et al. Association between performance measures and clinical outcomes for patients hospitalized with heart failure. JAMA 2007; 297: 61-70.

2. Wisløff U, Støylen A, Loennechen JP, et al. Superior cardiovascular effect of aerobic interval training versus moderate continuous training in heart failure patients: a randomized study. Circulation 2007; 115: 3086-94.

3. Flynn KE, Piña IL, Whellan DJ, et al. Effects of exercise training on health status in patients with chronic heart failure: HF-ACTION randomized controlled trial. JAMA 2009; 301: 1451-9.

4. Beckers PJ, Denollet J, Possemiers NM, Wuyts FL, Vrints CJ, Conraads VM. Combined endurance-resistance training vs. endurance training in patients with chronic heart failure: a prospective randomized study. Eur Heart J 2008; 29: 1858-66.

5. Roul G, Germain P, Bareiss P. Does the 6-minute walk test predict the prognosis in patients with NYHA class II or III chronic heart failure? Am Heart J 1998; 136: 449-57.

6. Davies EJ, Moxham T, Rees K, et al. Exercise training for systolic heart failure: Cochrane systematic review and meta-analysis. Eur J Heart Fail 2010; 12: 706-15. (Review)

7. Shah MR, Hasselblad V, Gheorghiade M, et al. Prognostic usefulness of the six-minute walk in patients with advanced congestive heart failure secondary to ischemic or nonischemic cardiomyopathy. Am J Cardiol 2001; 88: 987-93.

8. Alahdab MT, Mansour IN, Napan S, Stamos TD. Six minute walk test predicts long-term all-cause mortality and heart failure rehospitalization in African-American patients hospitalized with acute decompensated heart failure. J Card Fail 2009; 15: 130-5.

9. Tsutsui H, Tsuchihashi-Makaya M, Kinugawa S, Goto D, Takeshita A; JCARE-GENERAL Investigators. Characteristics and outcomes of patients with heart failure in general practices and hospitals. Circ J 2007; 71: 449-54.

10. Guidelines for Rehabilitation in Patients with Cardiovascular Disease (JCS 2007). Available at: http://www.j-circ.or.jp/guideline/ pdf/JCS2007_nohara_d.pdf. Accessed May 9, 2014.

11. ATS Committee on Proficiency Standards for Clinical Pulmonary Function Laboratories. ATS statement: guidelines for the sixminute walk test. Am J Respir Crit Care Med 2002; 166: 111-7.

12. Sung L, Hayden J, Greenberg ML, Koren G, Feldman BM, Tomlinson GA. Seven items were identified for inclusion when reporting a Bayesian analysis of a clinical study. J Clin Epidemiol 2005; 58: 261-8. (Review)

13. Lachin JM. Introduction to sample size determination and power analysis for clinical trials. Controlled Clinical Trails 1981; 2: 93113 .

14. Walker AM. Reporting the results of epidemiological studies. AM J Public Health 1986; 76: 556-8.

15. Bagley SC, White H, Golomb BA. Logistic regression in the medical literature: standards for use and reporting, with particular attention to one medical domain. J Clin Epidemiol 2001; 54: 97985.

16. Metz CE. Basic principles of ROC analysis. Semin Nucl Med 1978; 8: 283-98.

17. Cahalin LP, Mathier MA, Semigran MJ, Dec GW, DiSalvo TG. The six-minute walk test predicts peak oxygen uptake and survival in patients with advanced heart failure. Chest 1996; 110: 325-32.

18. Nogueira PA, Leal AC, Pulz C, Nogueira ID, Filho JA. Clinical reliability of the 6 minute corridor walk test performed within a week of a myocardial infarction. Int Heart J 2006; 47: 533-40.

19. Guyatt GH, Thompson PJ, Berman LB, et al. How should we measure function in patients with chronic heart and lung disease? J Chronic Dis 1985; 38: 517-24.

20. Lipkin DP, Scriven AJ, Crake T, Poole-Wilson PA. Six minute walking test for assessing exercise capacity in chronic heart fail- 
ure. Br Med J 1986; 292: 653-5.

21. Faggiano P, D'Aloia A, Gualeni A, Lavatelli A, Giordano A. Assessment of oxygen uptake during the 6-minute walking test in patients with heart failure. Am Heart J 1997; 134: 203-6.

22. Giannuzzi P, Temporelli PL, Corrà U, Tavazzi L; ELVD-CHF Study Group. Antiremodeling effect of long-term exercise training in patients with stable chronic heart failure: results of the Exercise in Left Ventricular Dysfunction and Chronic Heart Failure (ELVDCHF) Trial. Circulation 2003; 108: 554-9.

23. Martínez-Sellés M, García Robles JA, Prieto L, Domínguez Muñoa M, Frades E, Díaz-Castro O, Almendral J. Systolic dysfunction is a predictor of long term mortality in men but not in women with heart failure. Eur Heart J 2003; 24: 2046-53.

24. Chang AT, Seale H, Walsh J, Brauer SG. Static balance is affected following an exercise task in chronic obstructive pulmonary disease. J Cardiopulm Rehabil Prev 2008; 28: 142-5.

25. Moriello C, Finch L, Mayo NE. Relationship between muscle strength and functional walking capacity among people with stroke. J Rehabil Res Dev 2011; 48: 267-75.

26. Packer M, Bristow ML, Cohn JN, et al. The effect of carvedilol on morbidity and mortality in patients with chronic heart failure. U.S. Carvedilol Heart Failure Study Group. N Engl J Med 1996; 334 : 1349-55. 